\title{
INFORMALIDAD LABORAL EN LA FABRICACIÓN DE ROPA EN ARGENTINA ENTRE 2003-2018: ¿QUÉ APORTA LA ENCUESTA PERMANENTE DE HOGARES?
}

\author{
LABOUR INFORMALITY IN THE MANUFACTURE OF CLOTHING \\ IN ARGENTINA BETWEEN 2003-2018: WHAT DOES THE
}

PERMANENT HOUSEHOLD SURVEY CONTRIBUTE?

\author{
Gustavo Ludmer* \\ Federico Favata**
}

enviado: 06 de marzo 2019 - aceptado: 07 junio 2019

\begin{abstract}
Resumen
A nivel mundial, la fabricación de ropa se caracteriza por las adversas condiciones laborales que sufren sus trabajadores. Con un abordaje metodológico cuantitativo, este artículo analiza la relación entre los atributos observables tanto individuales como del puesto de trabajo y la informalidad laboral del sector en Argentina entre 2003 y 2018. Utilizando los microdatos de la Encuesta Permanente de Hogares, se realizó una descomposición Oaxaca-Blinder para conocer los determinantes observables de la problemática. Los resultados obtenidos sugieren que: i) los trabajadores del sector tienen mayor probabilidad de ser informales que el resto de los ocupados en Argentina; ii) dicha diferencia se explica principalmente por la mayor presencia relativa de trabajadores y puestos de trabajo con atributos usualmente penalizados en el mercado laboral; y iii) por una penalidad propia del sector, que aumenta las probabilidades de ser informal, aun controlando por dichos atributos.
\end{abstract}

Código JEL: J24, J46, J81.

Palabras clave: costureros, informalidad laboral, Oaxaca-Blinder.

Ludmer, G. \& Favata, F. (2020). Informalidad Laboral en la fabricación de ropa en argentina entre 2003-2018: ¿qué aporta la encuesta permanente de hogares? Estudios económicos, 37 (74), 105-126.

* Centro de Investigaciones y Estudios Laborales (CEIL). Correo electrónico: gusludmer@gmail.com

** Centro de investigación macroeconómica para el desarrollo (CIMaD). Correo electrónico: ffavata@ unsam.edu.ar 


\begin{abstract}
Globally, the manufacture of clothing is characterized by adverse working conditions suffered by its workers. With a quantitative methodological approach, this article analyzes the relationship between the observable attributes of both the individual and the workplace and the labor informality of the sector in Argentina between 2003 and 2018. Using microdata from the Permanent Household Survey, an Oaxaca-Blinder decomposition was carried out to determine the observable determinants of the problem. The results obtained suggest that: i) workers in the sector are more likely to be informal than the rest of those employed in Argentina; ii) this difference is mainly explained by the greater relative presence of workers and jobs with attributes usually penalized in the labor market; and, iii) by an intrinsic penalty of the sector, which increases the probability of being informal, even controlling for such attributes.
\end{abstract}

JEL Code: J24, J46, J81.

Keywords: sewing workers, labor informality, Oaxaca-Blinder. 


\section{INTRODUCCIÓN}

En la actualidad, la elevada incidencia de la informalidad laboral en la industria de fabricación de indumentaria resulta una constante a lo largo de todo el planeta (Esbenshade, 2004; OIT, 2016). En una dinámica que se repite tanto a nivel internacional como local, la mayoría de los ocupados de la rama perciben ingresos reducidos, no están cubiertos por las regulaciones laborales ni por las instituciones de la seguridad social (Montero Bressan, 2012).

Según información del Observatorio de Empleo y Seguridad Social (OEDE), durante 2017 la confección de prendas de vestir generó 44000 empleos asalariados formales en Argentina (Ludmer, 2018). A nivel nacional, el sector se caracteriza por una elevada tasa de informalidad laboral y la importancia del empleo por cuenta propia (Lieutier, 2010). Con los microdatos de la Encuesta Permanente de Hogares (EPH) del Instituto Nacional de Estadísticas y Censos (INDEC), es posible estimar la presencia de aproximadamente 162000 trabajadores en la rama durante 2017, lo que deja en evidencia su importancia en la generación de empleo en el país.

Diversos autores destacan que en Argentina la tasa de informalidad en esta industria ronda entre el $60 \%$ y el $70 \%$ del empleo, concentrándose el empleo informal en talleres clandestinos (Lieutier et al., 2018; OIT, 2013). Esas pequeñas unidades productivas suelen ser viviendas particulares que no tienen habilitación municipal, no cumplen con las normativas laborales ni impositivas ni de seguridad e higiene (Amengual, 2011). Se expandieron durante la década de 1990 con la crisis de la industria nacional: hacia el año 2008 un relevamiento estimaba la presencia de 5000 talleres clandestinos en la Ciudad de Buenos Aires y una cantidad similar en el Conurbano Bonaerense (Lieutier, 2010).

Particularmente en nuestro país, la informalidad laboral trae diversas consecuencias negativas para los trabajadores del sector, tales como ingresos laborales inferiores a los que establece el convenio colectivo (N. ${ }^{\circ} 626$ de 2011), falta de acceso al aguinaldo, a indemnización por despido, a vacaciones pagas, a Aseguradora de Riesgos del Trabajo (ART), a obra social y a aportes jubilatorios, entre otros perjuicios (OIT, 2015). Usualmente el pago se realiza por prenda terminada, lo que carga sobre el trabajador el riesgo de las oscilaciones de la demanda, característica particular del mercado de la ropa (Salgado, 2012).

En el presente trabajo se analiza la EPH del INDEC entre 2003 y 2018 con el objetivo de descubrir cuáles son los aportes que puede realizar para comprender la problemática. Dado que, como se verá más adelante, el sector emplea 
mayoritariamente a trabajadores que suelen ser informales (por ejemplo, mujeres e individuos de bajo nivel educativo), cabe preguntarse si la mayor informalidad se debe a que aglutina a dichos trabajadores o si, aun controlando por los atributos personales observables, el sector genera una penalidad propia adicional.

Para responder la pregunta general de la investigación, será necesario abordar los siguientes interrogantes específicos:

1. ¿Cuáles son las características observables de los ocupados y los puestos de trabajo en la confección de ropa que se destacan respecto al total del empleo de la economía argentina?

2. La diferencia en los niveles de informalidad laboral entre los ocupados del sector y del total de la economía, ¿es estadísticamente significativa? ¿Cómo evolucionó en el tiempo?

3. En caso de comprobarse diferencias estadísticamente significativas, ¿corresponden enteramente a los atributos observables de los ocupados y sus puestos de trabajo o se verifica la presencia de una penalidad propia del sector en materia de informalidad?

El período de tiempo del presente artículo fue elegido según la disponibilidad de microdatos para la modalidad continua de la EPH. Los 15 años comprendidos entre 2003 y 2018 permitirán comprobar si hubo modificaciones significativas en la problemática a lo largo del tiempo ${ }^{1}$.

Para responder la pregunta de investigación, en primer lugar se realiza una exhaustiva revisión bibliográfica que permitió detectar el área de vacancia en las investigaciones sobre las causas de la informalidad laboral. Luego se describe la metodología de abordaje cuantitativa utilizada. En la tercera sección se presentan los resultados del análisis. Por último, se plantean las principales conclusiones del estudio y se sugieren algunas líneas de investigación futuras.

1 Cabe aclarar que no se encuentran disponibles las bases del tercer trimestre de 2007, del tercero y cuarto de 2015 y el primero de 2016. 


\section{REVISIÓN DE LA LITERATURA}

\section{I.1. Estudios sobre informalidad laboral en indumentaria en el mundo}

A nivel mundial, diversos estudios destacan la precariedad de las condiciones laborales en esta industria desde fines del siglo XIX y principios del XX (Ross, 1997; Esbenshade, 2004, entre otros). La organización de los trabajadores costureros durante el modelo de acumulación fordista permitió una mejora notoria de las condiciones laborales durante las décadas posteriores a la Segunda Guerra Mundial en los países desarrollados (Green, 1997; Montero Bressan, 2012).

Desde la década de 1970 tuvo lugar una reconfiguración mundial de la producción de ropa que implicó su relocalización hacia países con menores salarios y la proliferación de talleres del sudor (sweatshops) en diversos países desarrollados (Green, 1997; Ross, 1997; Esbenshade, 2004; Montero Bressan, 2012). Robert Ross (2004) denomina "race to the bottom" (carrera al fondo) a la feroz competencia desatada entre talleristas de países pobres por ganar mercados, a través de la minimización de los costos de producción, entre los que se incluyen salarios, beneficios sociales e impuestos. Para Andrew Ross (1997), la relocalización de la producción motivó el comienzo de una espiral salarial descendente basada en la competencia entre los trabajadores de las maquilas, los obreros norteamericanos y los trabajadores inmigrantes en los sweatshops.

Respecto al rol de la inmigración, Bonacich et al. (2000) concluyen que el sistema de sweatshops es posible en Estados Unidos gracias a la presencia de inmigrantes latinos y asiáticos, muchos de ellos indocumentados, sin otras posibilidades de empleo producto de la segmentación racial que caracteriza dicho mercado laboral. La discriminación que sufren la mayoría de los inmigrantes restringe sus oportunidades laborales e incrementa su vulnerabilidad social, debilitando su poder de negociación para defender sus condiciones laborales, fenómeno también detectado en estudios para Argentina (por ejemplo, Lieutier, 2010).

Por último, el empleo femenino también resulta una constante en esta industria a nivel mundial. Shaw (2002) estima la presencia de alrededor de 35 millones de mujeres en el sector de indumentaria, de las cuales aproximadamente 26.6 millones tendrían empleos informales. 
I.2. Estudios sobre informalidad laboral en la fabricación de indumentaria en Argentina

Las causas de la informalidad laboral en la industria argentina de fabricación de ropa fueron abordadas por varios autores en los últimos años (Gallart, 2006; Lieutier, 2010; Amengual, 2011; Salgado, 2012; Lieutier et al., 2018, entre otros).

La industria de confección de ropa nació en Argentina hacia fines del siglo XIX y alcanzó su apogeo hacia mediados de la década de 1950, cuando empleó, junto con el eslabón textil, a 179000 trabajadores, el 14\% del empleo de la industria nacional (Canitrot et al., 1976). Entre 1976-1981 y 1991-2001, diversos gobiernos implementaron medidas de apertura comercial en un contexto de apreciación cambiaria (dólar barato) que condujo a un fuerte incremento de las importaciones de prendas (Kosacoff, 2004). Este fenómeno tuvo lugar en paralelo a la caída del poder adquisitivo de los trabajadores y el aumento de la desocupación, lo que deprimió la demanda doméstica de ropa. La combinación de ambas tendencias desató una intensa competencia entre las fábricas y los talleres que derivó tanto en destrucción como en precarización del empleo a niveles históricamente elevados (Montero Bressan, 2017).

Ferreira y Schorr (2012) destacan que la confección de ropa prácticamente no tiene barreras a la entrada pues para montar un taller de costura se necesita un capital mínimo (máquinas de bordar y coser cuyos precios rondan los U\$S 200). Asimismo, se trata de un oficio que tradicionalmente ha sido asignado a las mujeres, con lo cual existe una gran cantidad de trabajadoras capaces de realizarlo (Burchielli et al., 2014). Ambos factores determinan la existencia de una gran cantidad de talleres dispuestos a realizarlo, lo que genera una intensa competencia por las órdenes de trabajo. Esto conduce a una minimización del ingreso de los talleres, que impide la formalización de sus trabajadores (Lieutier, 2010).

Amengual (2011) sostiene que la elevada informalidad laboral en esta rama también es producto de una deficiente regulación de los distintos niveles del Estado (nacional, provincial y municipal), producto de la falta de voluntad política y de la dificultad para localizar las unidades productivas debido a su mínimo tamaño. Gallart (2006) aporta que la atomización de los trabajadores en los talleres clandestinos y en el trabajo a domicilio dificulta su sindicalización y la defensa colectiva de sus intereses.

La mayoría de los antecedentes en este campo son estudios cualitativos: existen pocos análisis que incluyen métodos cuantitativos. El artículo de Lieutier et al. (2018) utiliza los microdatos de la Encuesta Anual de Hogares Urbanos (EAHU) 
de la Ciudad Autónoma de Buenos Aires (CABA) del período 2012 y 2016 para elaborar una estadística descriptiva sobre el empleo en el sector. Los autores estiman que la informalidad laboral afectó al 56\% de los trabajadores costureros en la CABA. También destacan que más del $60 \%$ de los ocupados informales que viven en la Ciudad nacieron en otros países, tienen ingresos horarios $37 \%$ menores a los de los costureros formales con jornadas laborales que superan las 45 horas por semana y se emplean mayoritariamente en micro y pequeñas unidades productivas (Lieutier et al., 2018).

Carpio y Salgado (2017) calculan una tasa de informalidad laboral de 50\% para los asalariados de la industria de fabricación de indumentaria que viven en la Ciudad de Buenos Aires (aproximadamente 28 500), en función del análisis de una base mancomunada que junta microdatos de los años 2006, 2009, 2012 y 2015. Además destacan que el $38.5 \%$ de los ocupados de la rama nacieron en países limítrofes. Al interior de este colectivo, el $71.4 \%$ de los asalariados no poseen aportes a la seguridad social (mientras que para los trabajadores que nacieron en la CABA esa incidencia se reduce al 32.6\%). Un fenómeno similar ocurre para los ocupados con ingresos por debajo del salario mínimo ( $48 \%$ de los nacidos en países limítrofes y $18.6 \%$ para los oriundos de CABA) y para quienes trabajan más de 49 horas por semana (47.4\% vs. $23.6 \%$ ). Las adversas condiciones en materia de formalidad de los puestos, ingresos y horas trabajadas llevan a dichos autores a concluir que el concepto de precariedad laboral resulta insuficiente para captar la dimensión de la problemática y proponen usar el de superexplotación laboral (Carpio y Salgado, 2017).

\section{METODOLOGÍA}

A continuación se describen las fuentes de información utilizadas, la metodología para calcular la informalidad laboral y el tratamiento de la información para el análisis estadístico descriptivo y la descomposición Oaxaca-Blinder.

\section{II.1. Fuentes de información}

Se trabajó con los microdatos trimestrales de la EPH del INDEC que capta información de 31 aglomerados urbanos en Argentina, con una tasa de cobertura estadística de aproximadamente $62 \%$ de la población urbana (Zack et al., 2017). La EPH presenta limitaciones para trabajar con desagregación sectorial (a dos dígitos de nivel de actividad del Clasificador Internacional Industrial Uniforme, CIIU), pues no posee suficiente cantidad de observaciones por trimestre. Para agrandar la 
muestra se construyeron pools de datos de corte transversal juntando ondas trimestrales consecutivas de la EPH en línea con el tratamiento adoptado en Maurizio et al. (2008). También se consultó los datos sobre evolución de la producción nacional de ropa en el INDEC y de la cantidad de asalariados registrados en la seguridad social en el Ministerio de Producción y Empleo de la Nación.

\section{II.2. Medición de la informalidad}

A la hora de operacionalizar la formalidad de los puestos de trabajo, en el caso de los costureros asalariados el criterio adoptado consideró si tienen aportes jubilatorios (de su empleador o propios), en línea con los estudios de informalidad en Argentina con la EPH (por ejemplo, Beccaria y Groisman, 2008). Respecto a los trabajadores por cuenta propia y a los patrones, de gran importancia en este sector, se optó por definir su formalidad según su nivel educativo: aquellos con estudios universitarios completos (profesionales) fueron considerados formales mientras que al resto se los supuso informales, en línea con el criterio de Beccaria y Groisman (2008). Por último, los trabajadores familiares sin remuneración fueron incluidos dentro del universo de los informales.

La metodología utilizada para estimar la evolución de la tasa de informalidad laboral en el sector sigue el método desarrollado en Porta et al. (2016). Dicho trabajo estimó las tasas de informalidad laboral en asalariados para todos los sectores de la industria manufacturera argentina (a dos dígitos del CIIU). Dada la escasa cantidad de observaciones por trimestre, los autores construyen varios pools de datos de corte transversal integrados por las ondas trimestrales de cuatro años consecutivos para conocer las tasas de informalidad a dos dígitos (Porta et al., 2016). Una vez calculado el promedio de informalidad del sector para cada uno de los períodos cuatrianuales, asignan el comportamiento anual de la informalidad del conjunto de la industria ${ }^{2}$ siguiendo la fórmula:

\section{TI costura $2004=\underline{T I}$ costura 2003-2006 * (TI Industria 2004)}

TI Industria 2003-2006

Con TI costura 2004 como tasa de informalidad laboral en la confección de prendas de vestir para el año 2004 calculada como el producto entre la tasa de informalidad laboral en el sector para el período 2003-2006 y la tasa de informalidad labo-

2 Trabajar en términos anuales a nivel industria argentina es posible dado que posee la suficiente cantidad de casos. 
ral de la industria de 2004, dividido por la tasa de informalidad laboral de la industria para el mismo período 2003-2006. Dada la importancia del trabajo por cuenta propia en este sector, se realizó el cálculo de la informalidad laboral para todos los ocupados (y no solo para los asalariados como hicieron Porta et al. [2016]).

\section{II.3. Estadística descriptiva}

Se elaboró un ejercicio de estadística descriptiva para conocer los atributos observables tanto individuales como del puesto de trabajo que predominan en la industria de ropa, comparado con el empleo del resto de la economía argentina.

Para incrementar la muestra de costureros relavados se conformó un pool con las ondas de la EPH para el período comprendido entre el segundo trimestre de 2016 y el tercero de 2018 (último disponible al momento de la elaboración del artículo). Se trabajó con los casos expandidos por su respectivo coeficiente de expansión muestral.

\section{II.4. Descomposición Oaxaca-Blinder y modelo probit}

Con el objetivo de analizar la existencia de una mayor informalidad laboral solo por estar ocupado en la confección de prendas de vestir en Argentina, se realizó una descomposición Oaxaca-Blinder. Esta técnica se utiliza en economía laboral para detectar la existencia de discriminación hacia diversos grupos de trabajadores. Permite conocer si las diferencias en los salarios (por ejemplo) entre dos grupos se originan porque poseen distintos atributos (niveles educativos, por ejemplo) o si obedecen a la presencia de una discriminación salarial, ante los mismos atributos observables (Ospino et al., 2010).

Para ello, antes fue necesario realizar otro ejercicio econométrico: un modelo no lineal (probit) para explicar la probabilidad de un trabajador de ser informal según diversos atributos observables tanto individuales como del puesto de trabajo. En el modelo probit estimado, la variable dependiente adoptó valor uno si el trabajador fue informal y cero en caso contrario. Las variables explicativas utilizadas fueron: dummies de nivel educativo (la categoría base fue secundaria completa), edad, edad al cuadrado, dummies regionales (la categoría base fue Gran Buenos Aires), dummy de género, dummy de extranjero, dummy si el ocupado trabajó en su domicilio y dummies de tamaño del establecimiento productivo, medido en cantidad de ocupados (la categoría base fue un solo ocupado). 
Luego la técnica Oaxaca-Blinder permitió descomponer la brecha de informalidad promedio existente entre los costureros y el resto de los ocupados en tres efectos: i) el efecto dotaciones, que incluye las diferencias en las tasas de informalidad debido a los diferentes atributos observables que se explican por el modelo probit; ii) el efecto coeficientes, que condensa las diferencias no explicadas por el modelo, consideradas como una estimación de la discriminación laboral; y iii) el efecto interacción, que surge de la no trivialidad de la elección del grupo utilizado como base. La fórmula es:

$$
\begin{gathered}
y^{n c}-y^{c}=\Delta x \beta^{c}+\Delta \beta x^{n c}+\Delta x \Delta \beta \\
\Delta x=x^{n c}-x^{c} \& \quad \Delta \beta=\beta^{n c}-\beta^{c} \\
y^{n c}-y^{c}=E+C+C E
\end{gathered}
$$

Donde $y$ es la incidencia de la informalidad laboral, $x$ el vector de variables explicativas y $\beta$ los coeficientes. Los grupos definidos son los trabajadores costureros (C) y el resto de los ocupados de la economía (NC). E es el efecto dotación, $C$ es el efecto coeficiente y $C E$ es el efecto interacción.

Figura 1. Producción de ropa en Argentina: 2003-2018. En millones de pesos constantes de 2004

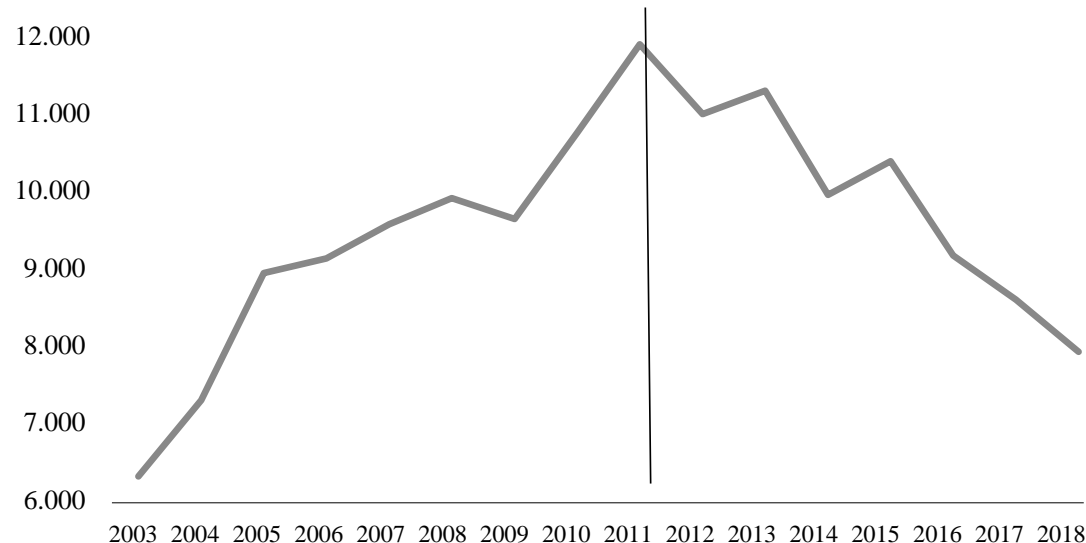

Fuente: elaboración propia sobre la base de datos de INDEC.

Para incrementar la muestra de costureros para el probit y el Oaxaca-Blinder, se elaboraron dos pools de datos: i) el primero abarcó entre el tercer trimestre de 2003 (cuando empieza el relevamiento continuo de la EPH) y el cuarto de 2011; ii) el segundo incluyó las ondas entre el primer trimestre de 2012 y el tercero de 2018. 
Los períodos temporales de cada pool fueron seleccionados según el comportamiento de la producción de ropa en Argentina. La figura 1 refleja que las cantidades fabricadas crecieron hasta el año 2011 (dinámica solo interrumpida durante 2009 por la crisis internacional), según información del INDEC. Desde entonces, la producción del sector cayó continuadamente hasta 2018.

\section{RESULTADOS OBTENIDOS}

III.1. Principales características individuales y del puesto de trabajo

La figura 2 resume los resultados del ejercicio de estadística descriptiva realizada con las ondas entre el segundo trimestre de 2016 y el tercero de 2018. Se trata de atributos personales y del puesto de trabajo especialmente seleccionados, como la participación en el empleo total de las mujeres, los extranjeros y los trabajadores de bajo nivel educativo, entre otros. La información se presenta tanto para los trabajadores del sector como para los ocupados totales del país, de forma tal de contar con un punto de comparación.

Figura 2. Características individuales y del puesto de trabajo en Argentina: 2016 a 2018. En \% del empleo total del sector

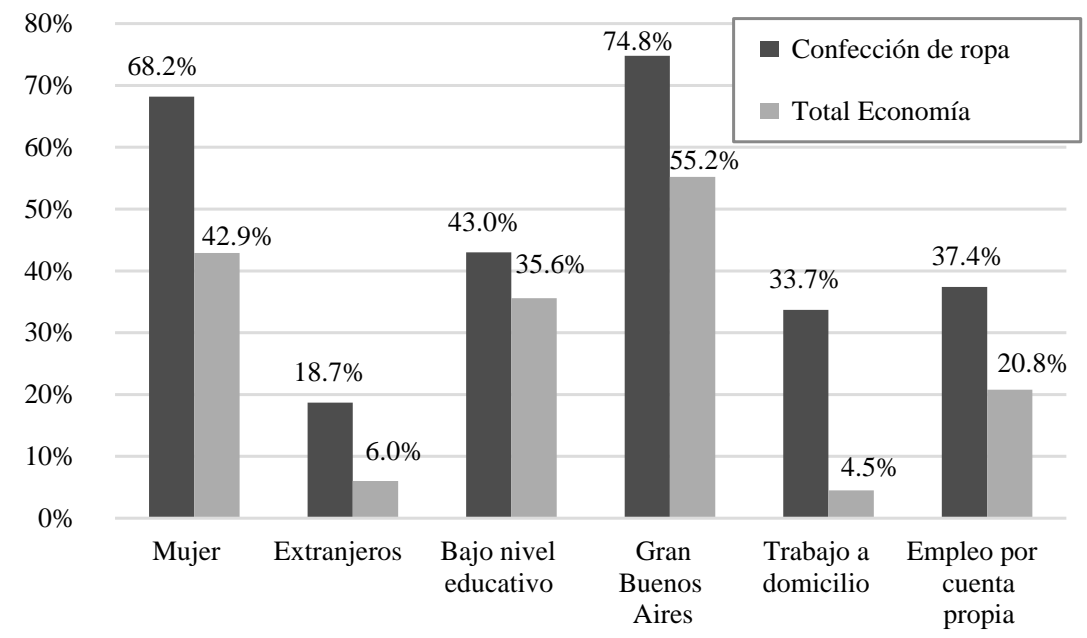

Fuente: elaboración propia en base a datos EPH de INDEC.

En primer lugar, los trabajadores del sector relevados por la EPH fueron mayoritariamente mujeres: $68.2 \%$ versus $42.9 \%$ del total de la economía. También 
se destacó la presencia de ocupados que nacieron en otros países (18.7\%), proporción que representa más del triple de los ocupados totales (6\%). En su interior, la colectividad boliviana fue la más numerosa, seguida por los trabajadores nacidos en Paraguay y Perú3.

Los trabajadores de bajo nivel educativo (hasta secundario incompleto) representaron el $43 \%$ del total en la fabricación de prendas, proporción que se redujo a $35.6 \%$ para la economía total. Otra característica particular del sector fue su excesiva concentración geográfica en el Gran Buenos Aires (GBA): el 74.8\% de los ocupados viven en la Ciudad de Buenos Aires y en el Conurbano Bonaerense, mientras que para los ocupados totales dicha participación fue de $55.2 \%{ }^{4}$.

El trabajo a domicilio resulta un fenómeno frecuente en el sector y se asocia a estrategias de tercerización de la confección de prendas por parte de talleres, fábricas y marcas (OIT, 1998). Mientras que para la economía en su conjunto solo $4.5 \%$ de los trabajadores se desempeñaron en su domicilio, en este sector dicha proporción fue de $33.7 \%$.

Por último, se destacó la elevada presencia de trabajadores por cuenta propia (independientes): $37.4 \%$, versus el $20.8 \%$ de la economía argentina. Estas particularidades propias de los trabajadores del sector cobran especial importancia a la hora de explicar la problemática de la informalidad laboral, como se verá más adelante.

\section{III.2. Evolución de la informalidad}

La figura 3 muestra la evolución de la tasa de informalidad laboral en la confección de prendas en Argentina desde 2003 a 2018, calculada siguiendo la metodología de Porta et al. (2016).

Entre 2003 y 2013, la informalidad laboral en la confección pasó de 83\% de los trabajadores a $67.5 \%$, lo que representó una reducción de 15.5 puntos porcentuales (o 18.7\%). En primer lugar, conviene señalar que esta dinámica sectorial se contextualiza dentro de la tendencia general del mercado de trabajo argentino, donde la informalidad para el total de los ocupados pasó de 55.9\% en 2003 a $42.1 \%$ en 2014 (una reducción de $24.7 \%$, superior que en el sector de confección). En

3 Según la EPH, $60 \%$ de los costureros extranjeros fueron bolivianos, $21 \%$ paraguayos y $11 \%$ peruanos.

4 La EPH, al relevar únicamente aglomerados urbanos, sobreestima la importancia del GBA en el empleo total del país. 
este sentido, el crecimiento económico experimentado en esos años permitió la recuperación del empleo y del poder de negociación de trabajadores y sindicatos, lo que permitió la formalización de una parte de los puestos de trabajo informales así como la creación de nuevos puestos registrados, disminuyendo la tasa de informalidad (Porta et al., 2016).

Figura 3. Tasa de informalidad laboral en la fabricación de ropa en Argentina: 2003 a 2018. En \% del empleo total del sector

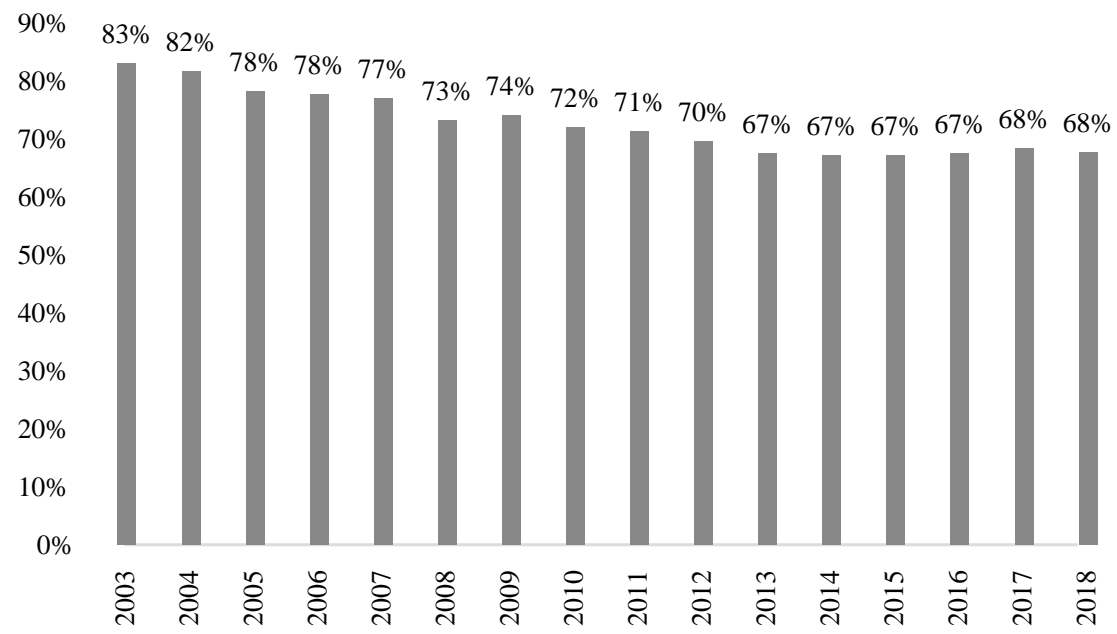

Fuente: elaboración propia en base a EPH de INDEC.

La caída de la informalidad en el sector tuvo lugar en años donde la producción de ropa mostró un intenso crecimiento (hasta fines de 2011). Esto apoya la hipótesis de que en un contexto expansivo los trabajadores lograron mejoras en las condiciones laborales. Lamentablemente, la información estadística disponible no permite diferenciar si el crecimiento del sector fue traccionado por los eslabones productivos de mayor formalidad laboral o por los más informales.

Desde 2013, la tasa de informalidad en el sector se estabilizó en torno a $68 \%$ del empleo total, dejando en evidencia la existencia de un núcleo duro difícil de perforar. Se verifica que dicha estabilización tuvo lugar en un marco de caída de la producción de ropa y destrucción de puestos de trabajo, lo que deja en evidencia que la contracción afectó en proporciones equivalentes al empleo registrado y al no registrado. 


\section{III.3. Resultados del Modelo}

La tabla 1 resume los resultados obtenidos para el modelo probit estimado para conocer las causas de la informalidad laboral en Argentina. Trabajar en la costura incrementa entre $9.3 \%$ y $5.3 \%$ la probabilidad de ser informal en ambos períodos, controlando por otros atributos observables. Esto muestra la presencia de una penalidad en informalidad para los ocupados del sector.

Tabla 1. Resultados del modelo probit. Períodos 2003-2011 y 2012-2018

\begin{tabular}{lcc}
\hline & $2003-2011$ & $2012-2018$ \\
\cline { 2 - 4 } & informal & informal \\
\hline Educación hasta primaria incompleta & $.3188^{* * *}$ & $.2993^{* * *}$ \\
\hline Educación primaria completa & $.2205^{* * *}$ & $.1920^{* * *}$ \\
\hline Educación secundaria incompleta & $.1604^{* * *}$ & $.1478^{* * *}$ \\
\hline Educación superior incompleta & $-.0268^{* * *}$ & $-.0287^{* * *}$ \\
\hline Educación superior completa & $-.4585^{* * *}$ & $-.4324^{* * *}$ \\
\hline Edad & $-.0365^{* * *}$ & $-.0355^{* * *}$ \\
\hline Edad al cuadrado & $.0004^{* * *}$ & $.0004^{* * *}$ \\
\hline Región NOA & $.1513^{* * *}$ & $.1480^{* * *}$ \\
\hline Región NEA & $.0890^{* * *}$ & $.0844^{* * *}$ \\
\hline Región Cuyo & $.1358^{* * *}$ & $.1086^{* * *}$ \\
\hline Región Pampeana & $.0061^{* * *}$ & .0004 \\
\hline Región Patagonia &. $.1359^{* * *}$ & $-.1066^{* * *}$ \\
\hline Mujer
\end{tabular}




\begin{tabular}{|c|c|c|}
\hline Tamaño 4: 11 a 40 individuos & $-.2157 * * *$ & $-.2164 * * *$ \\
\hline Tamaño 5: 41 a 200 individuos & $-.3703 * * *$ & $-.3314 * * *$ \\
\hline Tamaño 6: más de 200 individuos & $-.3505 * * *$ & $-.3331 * * *$ \\
\hline Observations & 728,378 & 566,227 \\
\hline
\end{tabular}

Fuente: elaboración propia sobre la base de EPH-INDEC.

La tabla 2 presenta los resultados de la descomposición Oaxaca-Blinder.

Tabla 2. Resultados de la descomposición Oaxaca-Blinder. Períodos 2003-2011 y 2012-2018

\begin{tabular}{ccc}
\hline & $2003-2011$ & $2012-2018$ \\
\hline Diferencias & $-.2914 * * *$ & $-.2662 * * *$ \\
\hline Dotaciones & $-.2489 * * *$ & $-.2502^{* * *}$ \\
\hline Coeficientes & $-.0478^{* * *}$ & $-.0316^{* * *}$ \\
\hline Interacción & .0052 & $.0156^{* *}$ \\
\hline$* * *$ Significativo al $99 \%, * * 95 \% \mathrm{y} *$ al $90 \%$ & & \\
\hline
\end{tabular}

Fuente: elaboración propia sobre la base de EPH-INDEC.

En los dos períodos analizados, se destaca la presencia de una mayor probabilidad de ser informal entre los trabajadores del sector de confección de indumentaria que en el resto de la población ocupada (significativamente distinta de cero). En efecto, dado que el grupo base utilizado fue la población ocupada general, el signo negativo de las diferencias indica que los costureros fueron informales en mayor proporción.

El efecto dotaciones, que captura las diferencias en la informalidad provenientes de distintos atributos observables en cada grupo, explica el $85.4 \%$ de la diferencia observada en el primer período y el $94 \%$ en el segundo. El efecto coeficientes, que captura la distinta forma en que dichos atributos son penalizados en la costura, explica el 16.6\% y el 11.9\% de la diferencia respectivamente. Ambos efectos son estadísticamente distintos de cero para cualquier nivel de confianza. Por último, el efecto interacción es significativamente distinto de cero únicamente para el segundo período, explicando el $5.9 \%$ de las diferencias. 
En este punto, es posible realizar una primera conclusión: la informalidad laboral en el sector está principalmente asociada a la sobrerepresentación de ocupados con características individuales y de puestos de trabajo que suelen estar afectados por la problemática en el mercado laboral argentino. En segunda medida, también se detecta la existencia de una penalidad propia del sector de confección de ropa con cómo dichos atributos están afectados por una mayor informalidad laboral. Adicionalmente, queda en evidencia que la magnitud de la diferencia con el total de la economía prácticamente no se modificó en los dos períodos analizados: solo se redujo $8.6 \%$.

Para desagregar los determinantes de los efectos anteriores, la tabla 3 presenta los resultados de la descomposición según las distintas variables analizadas.

Tabla 3. Resultados desagregados de la descomposición Oaxaca-Blinder. Períodos 2003-2011 y 2012-2018

\begin{tabular}{|c|c|c|c|c|}
\hline & \multicolumn{2}{|c|}{ 2003-2011 } & \multicolumn{2}{|c|}{$2012-2018$} \\
\hline & Dotaciones & Coeficientes & Dotaciones & Coeficientes \\
\hline Educación primaria incompleta & .0004 & $.0030 * *$ & .0004 & $.0038^{* * *}$ \\
\hline Educación primaria completa & $-.0025 * * *$ & $.0199 * * *$ & .0004 & $.0231 * * *$ \\
\hline Educación sec. incompleta & -.0010 & $.0134 * * *$ & -.0002 & $.0137 * * *$ \\
\hline Educación superior incompleta & .0008 & $-.0032 * *$ & .0000 & -.0027 \\
\hline Educación superior completa & $-.0602 * * *$ & $.0080 * * *$ & $-.0577 * * *$ & $.0237 * * *$ \\
\hline Edad & $.0385 * * *$ & $-.3154 * * *$ & $.0362 * * *$ & $-.3398 * * *$ \\
\hline Edad al cuadrado & $-.0405 * * *$ & $.1325 * * *$ & $-.0384 * * *$ & $.1339 * *$ \\
\hline Región NOA & -.0003 & $.0134 * * *$ & .0009 & $.0087 * *$ \\
\hline Región NEA & $.0015^{* *}$ & $-.0102 * * *$ & .0001 & .0030 \\
\hline Región Cuyo & .0005 & $.0030 * *$ & -.0000 & $.0050 * *$ \\
\hline Región Pampeana & .0003 & -.0014 & -.0005 & .0021 \\
\hline Región Patagonia & $-.0079 * * *$ & .0014 & -.0011 & -.0021 \\
\hline Mujer & $-.0285 * * *$ & .0011 & $-.0150 * * *$ & .0057 \\
\hline Extranjero & $-.0058 * * *$ & $-.0024 *$ & $-.0124 * * *$ & $-.0090 * * *$ \\
\hline Tamaño 1: Un sólo individuo & $-.0607 * * *$ & $.0196 * * *$ & $-.0682 * * *$ & $.0268 * *$ \\
\hline
\end{tabular}


INFORMALIDAD LABORAL EN LA FABRICACIÓN DE ROPA EN ARGENTINA ENTRE 2003-2018...

\begin{tabular}{ccccc}
\hline Tamaño 2: 2 a 5 individuos & $-.0035^{* * *}$ & $-.0101^{* * *}$ & $-.0021^{*}$ & -.0050 \\
\hline Tamaño 3: 6 a 10 individuos & $-.0008^{* *}$ & $-.0085^{* * *}$ & $.0007^{*}$ & $-.0039^{* * *}$ \\
\hline Tamaño 4: 11 a 40 individuos & $-.0018^{* *}$ & $-.0065^{* * *}$ & $-.0065^{* * *}$ & -.0019 \\
\hline Tamaño 5: 41 a 200 individuos & $-.0167 * *$ & $.0030^{* * *}$ & $-.0176^{* * *}$ & $.0045^{* *}$ \\
\hline Tamaño 6: + de 200 individuos & $-.0133^{* * *}$ & .0006 & $-.0190 * * *$ & $.0026^{*}$ \\
\hline Trabaja en domicilio & $-.0474 * *$ & $-.0294 * * *$ & $-.0504 * * *$ & $-.0413^{* * *}$ \\
\hline$* * *$ Significativo al 99\%, ** 95\% y* al 90\% & \\
\hline
\end{tabular}

Fuente: elaboración propia sobre la base de EPH-INDEC.

En relación al efecto dotaciones, se observa una contribución significativa y negativa de educación superior completa, edad al cuadrado, regiones NOA y Cuyo, mujer, extranjero, tamaño del establecimiento y trabajador a domicilio. Los costureros son mayoritariamente mujeres, extranjeros y se desempeñan en micro y pequeñas empresas o desde sus domicilios, atributos que el mercado laboral penaliza con una mayor informalidad laboral. Es por ello que su mayor presencia relativa en el colectivo de trabajadores de la confección contribuye a explicar la mayor incidencia de la problemática.

A su vez, se verifica un aporte significativo de la mayor edad que caracteriza a los costureros a reducir la problemática. Los efectos de la edad y de la edad al cuadrado tienen signos distintos, lo que estaría reflejando la presencia de una relación con forma de parábola cóncava entre la edad y la probabilidad de informalidad del ocupado. Por último, dado que la mayor proporción de los trabajadores del sector vive en el GBA, ninguna de las regiones realiza un aporte de magnitud a la informalidad del grupo (menos del 1\% de probabilidad) y los signos se compensan entre ellas.

Con relación al efecto coeficientes de la descomposición Oaxaca-Blinder, se destaca que la mayoría de las variables tienen un efecto significativo, lo que deja en evidencia que el sector de confección de indumentaria penaliza de forma distinta al promedio de la economía.

Las variables educativas exhiben una contribución significativa de signo positivo en ambos períodos (a excepción de educación superior incompleta). Esto significa que la educación está mejor remunerada (a través de una mayor formalidad) en la fabricación de prendas de vestir respecto al total de la economía. 
En ambos períodos, la edad está significativamente penalizada en el sector, lo que incrementa la brecha de informalidad en torno a 32.7 puntos porcentuales en promedio. Este hallazgo del trabajo deja en evidencia que en la confección de indumentaria no aplica la tradicional trayectoria laboral de los ocupados de la economía, por la cual a mayor edad, mayor formalidad. La magnitud del efecto de esta variable conduce a la necesidad de posteriores estudios que investiguen la existencia de una trayectoria hacia la informalidad laboral para los trabajadores de mayor edad.

La variable dummy de género no posee una penalidad significativa en el sector a la hora de incrementar la probabilidad de ser informal, lo que deja en evidencia que la discriminación de género en materia de informalidad no obedece al propio sector de costura, sino a la dinámica más general del mercado de trabajo.

La dummy de nacionalidad muestra un valor pequeño, negativo y solo significativo al $90 \%$ de confianza para el período comprendido entre 2003 y 2011 , mientras que para el segundo período se torna significativo al $1 \%$ y explica una penalidad de 0.9 puntos porcentuales adicionales. Esto deja en evidencia el surgimiento de una penalidad por ser inmigrante particular en el sector de confección, en particular en épocas de contracción del nivel de actividad.

Adicionalmente, la dummy de trabajo a domicilio muestra una penalidad significativa y de entre 2.9 y $4.1 \%$, lo que deja en evidencia que además de la penalización más amplia que tiene el trabajo a domicilio, esa problemática se amplifica en el sector. Por último, existe una pequeña penalidad adicional para los trabajadores que se desempeñen en establecimientos productivos de hasta 40 trabajadores.

\section{CONCLUSIONES}

El objetivo del presente artículo fue responder la pregunta de si trabajar en la confección de ropa en Argentina incrementa las probabilidades de tener un empleo informal. A partir de realizar la descomposición Oaxaca-Blinder a la información de la EPH para el período 2003-2018, el presente trabajo comprobó lo siguiente:

a) existen diferencias estadísticamente significativas en la tasa de informalidad laboral en la fabricación de ropa y la economía argentina;

b) dichas diferencias prácticamente no se modificaron con el paso del tiempo; 
c) la mayor incidencia de la informalidad entre los costureros corresponde principalmente al hecho de que el sector emplea en mayor proporción (entre $85 \mathrm{y}$ 94\% según el período) a grupos de individuos también afectados por dicha problemática en el total de la economía. Es decir, a la mayor presencia relativa de atributos individuales y del puesto de trabajo (captado en el efecto dotaciones de la descomposición Oaxaca-Blinder) que tradicionalmente son penalizados en el mercado laboral. Por ejemplo, la mayor presencia de mujeres, trabajadores con bajo nivel educativo y el mayor porcentaje de inmigrantes;

d) adicionalmente, uno de los hallazgos originales del artículo es confirmar la existencia de una penalidad adicional en materia de informalidad que genera trabajar en el sector de fabricación de ropa para algunos atributos individuales, principalmente la edad (y, en menor medida, la nacionalidad). Este fenómeno (reflejado en el efecto coeficientes del Oaxaca-Blinder) indica que los trabajadores con mayor edad (y las mujeres y los inmigrantes) tienen una mayor penalidad en materia de informalidad solo por el hecho de desempeñarse en la fabricación de ropa, en relación al promedio de la economía argentina.

De esta forma, resulta posible afirmar que la fabricación de ropa actúa como un sector refugio para los trabajadores más vulnerables del mercado laboral argentino. Probablemente este fenómeno se explique por las reducidas barreras a la entrada que caracteriza a la actividad de confección, como por ejemplo la escasa necesidad de inversión de capital inicial (una máquina de coser), el tratarse de un oficio ya conocido por una gran cantidad de trabajadoras y la posibilidad de realizarlo desde el propio domicilio, entre otros factores.

Estos hallazgos dejan en evidencia la importancia del sector de confección de ropa en Argentina a la hora de generar empleo para trabajadores vulnerables y la necesidad de desarrollar políticas públicas que logren reducir los perjuicios asociados a la informalidad y a la precariedad de sus puestos de trabajo. En este contexto, la presente investigación busca constituirse en un insumo para el diseño de políticas públicas que busquen morigerar los efectos negativos del sector de fabricación de ropa sobre la informalidad de sus ocupados.

Por último, los resultados del artículo plantean posibles líneas de investigación futuras. En particular, resulta de interés reiterar el presente ejercicio para otros sectores de actividad, de forma tal de conocer cuáles ramas de actividad aminoran la problemática de la informalidad laboral en Argentina y cuáles, al igual que la fabricación de prendas, la incrementan. 


\section{REFERENCIAS BIBLIOGRÁFICAS}

Amengual, M. (2011). Cambios en la capacidad del Estado para enfrentar las violaciones de las normas laborales: los talleres de confección de prendas de vestir en Buenos Aires. Desarrollo Económico, 100 (202), 291-311.

Beccaria, L. \& Groisman, F. (2008). Informalidad y pobreza en Argentina. Revista Investigación económica, 67, 135-169. Recuperado de http://www.scielo. org.mx/pdf/ineco/v67n266/v67n266a5.pdf

Bonacich, E., Appelbaum, R. P. \& Appelbaum, R. (2000). Behind the label: Inequality in the Los Angeles apparel industry. Berkeley: University of California Press.

Burchielli, R., Delaney, A. \& Goren, N. (2014). Garment homework in Argentina: Drawing together the threads of informal and precarious work. The Economic and Labour Relations Review, 25 (1), 63-80.

Canitrot, A., Fidel, J., Juillerat, M. \& Lucángeli, J. (1976). El empleo en la industria textil Argentina. Análisis de comportamiento y de elección tecnológica. Desarrollo Económico, 16 (63), 349-371.

Carpio, J. \& P. Salgado (2015.) Superexplotación, Informalidad y Precariedad. Reflexiones a partir del trabajo en la industria de la confección. Revista Estudios del Trabajo, 54, 55-89. Recuperado de https://www.aset.org.ar/ docs/Dinorah\%20y\%20Carpio.pdf

Esbenshade, J. (2004). Monitoring Sweatshops: Workers, Consumers, and the Global Apparel Industry. Philadelphia: Temple University Press.

Ferreira, E. \& Schorr, M. (2012). La industria textil y de indumentaria en la Argentina. Informalidad y tensiones estructurales en la pos-convertibilidad. En M. Schorr (Comp.), Argentina en la pos-convertibilidad: ¿desarrollo o crecimiento industrial? (pp. 219-253). Buenos Aires: Miño y Dávila.

Gallart, M. A. (2006). Análisis de las estrategias de acumulación y supervivencia de los trabajadores ocupados en la rama de textiles y confecciones. En OIT (Ed.), Informalidad, pobreza y salario mínimo. Programa Nacional de Trabajo Decente: Argentina 2004-2007 (pp. 127-182). Buenos Aires: OIT.

Green, N. (1997). Ready-to-wear and ready-to-work. North Carolina: Duke University Press.

Kosacoff, B. (2004). Evaluación de un escenario posible y deseable de reestructuración y fortalecimiento del Complejo Textil argentino. Buenos Aires: Oficina de la Comisión Económica para América Latina. Recuperado de https://www.cepal.org/es/publicaciones/28466-evaluacion-un-escenario-posible-deseable-reestructuracion-fortalecimiento

Lieutier, A. (2010). Esclavos. Los trabajadores costureros de la Ciudad de Buenos Aires. Buenos Aires: Retórica Ediciones. 
Lieutier, A., Degliantoni, C. \& Morillas, S. (2018). La tercerización en el sector de indumentaria: su impacto en las condiciones de trabajo en la Ciudad de Buenos Aires. Trabajo presentado en el Seminario internacional sobre tercerización laboral organizado por la Facultad Latinoamericana de Ciencias Sociales, Buenos Aires.

Ludmer, G. (2018). Distribución de la renta en la cadena de valor de indumentaria en Argentina durante 2016. H-industri@: Revista de historia de la industria, los servicios y las empresas en América Latina, 12 (23), 91-114. Recuperado de http://ojs.econ.uba.ar/ojs/index.php/H-ind/article/view/1228

Maurizio, R., Perrot, B. \& Villafañe, S. (2008). Dinámica de la pobreza y el mercado de trabajo en Argentina post-convertibilidad. Buenos Aires: Ministerio de Trabajo, Empleo y Seguridad Social de la Nación. Recuperado de http:// www.trabajo.gob.ar/downloads/biblioteca_estadisticas/toe6_05-dinamica. pdf

Montero Bressan, J. (2012). La moda neoliberal: El retorno de los talleres clandestinos de costura. Geograficando, 8 (8), 19-37.

Montero Bressan, J. (2017). El Origen del Sistema de "Talleres del Sudor" en Buenos Aires. Trabajo presentado en $6^{\circ}$ Jornadas de Historia de la Industria y los Servicios organizado por el Área de Estudios de Industria Argentina y Latinoamericana, Buenos Aires. Recuperado de http://www.aesial.com/ uploads/1/1/2/4/11241421/vi_jhis_montero.pdf

OIT (1998). El trabajo a domicilio en Argentina. En Cuestiones de Desarrollo: documento de discusión Nro. 27. Santiago de Chile: OIT.

OIT (2013). Informalidad laboral en Argentina: segmentos críticos y políticas para la formalización. Buenos Aires: OIT. Recuperado de https://www.ilo.org/ buenosaires/publicaciones/WCMS_234705/lang--es/index.htm

OIT (2015). Migraciones laborales en Argentina: protección social, informalidad y heterogeneidades sectoriales. Buenos Aires: OIT. Recuperado de https:// www.ilo.org/buenosaires/publicaciones/WCMS_379419/lang--es/index.htm

OIT (2016). Redistributing value added towards labour in apparel supply chains: tackling low wages through purchasing practices. Miller, D. y Hohenegger, K. Conditions of Work and Employment Nro. 83. Ginebra: OIT. Recuperado de https://www.ilo.org/wcmsp5/groups/public/---ed_protect/---protrav/---travail/documents/publication/wcms_534536.pdf

Ospino Hernández, C., Roldán Vásquez, P., \& Barraza Narváez, N. (2010). La descomposición salarial de Oaxaca-Blinder: Métodos, críticas y aplicaciones. Una revisión de la literatura. Revista de Economía del Caribe, 5, 237-274. Recuperado de http://www.scielo.org.co/pdf/ecoca/n5/n5a06.pdf

Porta, F., Santarcángelo, J., \& Schteingart, D. (2016). Producción y empleo en el sector industrial argentino: 1998-2014.H-industri@: Revista de historia 
de la industria, los servicios y las empresas en América Latina, 10 (19), 101-139. Recuperado de http://ojs.econ.uba.ar/ojs/index.php/H-ind/article/ view/933

Ross, A. (1997). Introduction. En A. Ross (Ed.). No sweat: Fashion, free trade, and the rights of garment workers (9-37). New York: Verso.

Ross, R. (2004). Slaves to fashion: Poverty and abuse in the new sweatshops. Ann Arbor: The University of Michigan Press.

Salgado, P. (2012). El trabajo en la industria de la indumentaria: una aproximación a partir del caso argentino. Revista Trabajo y Sociedad, (18), 59-68. Recuperado de http://www.scielo.org.ar/scielo.php?script=sci_arttext\&pid=S1514-68712012000100004

Shaw, L. (2002). Refashioning Resistance: Women workers organizing in the global garment industry. Manchester: Women Working Worldwide.

Zack, G., Schteingart, D. \& Favata, F. (2017). Pobreza e indigencia en Argentina (2003-2017): construcción de una serie completa y metodológicamente homogénea. Trabajo presentado en la $52^{\circ}$ Reunión Anual organizada por la Asociación Argentina de Economía Política, Bariloche.

(C) 2020 por los autores; licencia no exclusiva otorgada a la revista Estudios económicos. Este artículo es de acceso abierto y distribuido bajo los términos y condiciones de una licencia Atribución-No Comercial 4.0 Internacional (CC BY-NC 4.0) de Creative Commons. Para ver una copia de esta licencia, visite http:// creativecommons.org/licenses/by-nc/4.0 